\title{
Acoustical characteristic predictions of a multi-layer system of a submerged vehicle hull mounted sonar simplified to an infinite planar model
}

\author{
Sung-Hee Kim ${ }^{1}$, Suk-Yoon Hong ${ }^{1}$, Jee-Hun Song ${ }^{2}$, Hyun-Gwon Kil ${ }^{3}$ \\ Jae Jin Jeon ${ }^{4}$ and Young-Soo $\mathrm{Seo}^{4}$ \\ ${ }^{I}$ Seoul National University, Seoul, Korea \\ ${ }^{2}$ Chonnam National University, Chonnam, Korea \\ ${ }^{3}$ University of Suwon, Gyeonggi, Korea \\ ${ }^{4}$ Agency for Defense Development, Gyeongnam, Korea
}

\begin{abstract}
Hull Mounted Sonar (HMS) is a long range submerged vehicle's hull-mounted passive sonar system which detects low-frequency noise caused by machineries of enemy ships or submerged vehicles. The HMS needs a sound absorption /insulation multi-layer structure to shut out the self-noise from own machineries and to amplify signals from outside. Therefore, acoustic analysis of the multi-layer system should be performed when the HMS is designed. This paper simplified the HMS multi-layer system to be an infinite planar multi-layer model. Also, main excitations that influence the HMS were classified into mechanical, plane wave and turbulent flow excitation, and the investigations for each excitation were performed for various models. Stiffened multi-layer analysis for mechanical excitation and general multi-layer analysis for turbulent flow excitation were developed. The infinite planar multi-layer analysis was expected to be more useful for preliminary design stage of HMS system than the infinite cylindrical model because of short analysis time and easiness of parameter study.
\end{abstract}

KEY WORDS: Hull Mounted Sonar (HMS); Infinite planar multi-layer structure; Mechanical force; Plane wave; Turbulent flow noise.

\section{INTRODUCTION}

Hull Mounted Sonar (HMS) system is a long range passive detector that is typically installed to both sides of a submerged vehicle. It detects low frequency noise ranges caused by the mechanical equipments of other submerged vehicles or ships. The HMS possesses a multi-layer structure that block noise from own vessel machineries and are reduces reflected noise of exterior signals such as a ping of enemies. Simultaneously, the multi-layer structure must provide precise measurements at certain frequency ranges. Because of these complex conditions, various HMS multi-layer combinations and arrangements must be accounted for during the design stages. Thus, a multi-layer analysis must be conducted in order to predict the multi-layer acoustic performance during the HMS preliminary design step.

However, testing every multi-layer case is a nearly impossible task. Furthermore, commonly used tools, such as those based on the finite element method or boundary element method, do not serve as appropriate alternatives for predicting acoustic characteristics of the multi-layer. Universal tools used for finite element analysis or boundary element analysis can be applied to the analysis of multi-layers. However, in order to properly apply these tools, specific HMS geometry is first needed. Unfortunately, 
the necessary HMS geometry is typically not available during the early design stage in which the tools may be applied. Investigating multi-layer system at high frequencies with commonly used tools is time and cost consuming. Confidentiality and proprietary regulations limit the availability of open HMS research.

A relatively large submerged vehicle containing a low-curvature pressure hull can be equipped with the HMS system. In addition, the HMS system is long enough to be simplified to an infinite system. This paper simplified the HMS multi-layer system to be an infinite planar multi-layer model. Skelton and James (1997) and Ko, Pyo and Seong (2001) developed infinite planar multi-layer theories for underwater structures. Kang, Seo, Shin and Jeon (2011) showed that the infinite planar theory agreed well with measured data acquired from acoustic windows. Stiffeners were applied to a thin plate by Evseev (1973) and Mace (1980), but not to a multi-layer structure. Ko and Schloemer (1989), Ko and Nuttall (1991) investigated two- and threelayer structures for turbulent flow noise, and a point hydrophone was assumed to be embedded within an elastomer layer. However, this approach does not facilitate the analysis of a general multi-layer model, and it cannot be applied to a model of a hydrophone in a fluid layer.

This paper presents a prediction method that can be applied to the HMS preliminary design stage. The method predicts acoustic characteristics of the HMS multi-layer system using the infinite planar theory. Thus, the main excitation types that affect the HMS system are classified, and the acoustic characteristics of the various multi-layer models are evaluated for each excitation type. A stiffened thin planar theory was extended to the elastic theory and applied to the multi-layer analysis. Turbulent flow noise analysis was performed on a general model including the case of a hydrophone in acoustic fluid layer.

\section{GOVERNING EQUATION OF AN INFINITE PLANAR MULTI-LAYER}

Skelton and James (1997) presented a multi-layer system analysis method that employed continuous conditions at the boundaries of a layer. He derived matrix relationships that satisfied continuous displacement and stress conditions of an isotropic elastic layer, and satisfied continuous displacement and pressure conditions of an acoustic fluid layer for three directions of rectangular coordinates at the upper and lower surfaces of a layer. He obtained a relation for the stiffener matrix, displacement matrix, and force matrix from the derived matrix relationships. The governing matrix equation for a multi-layer system was assembled using relation matrices of isotropic elastic layers and acoustic fluid layers.

\section{Acoustic fluid layer}

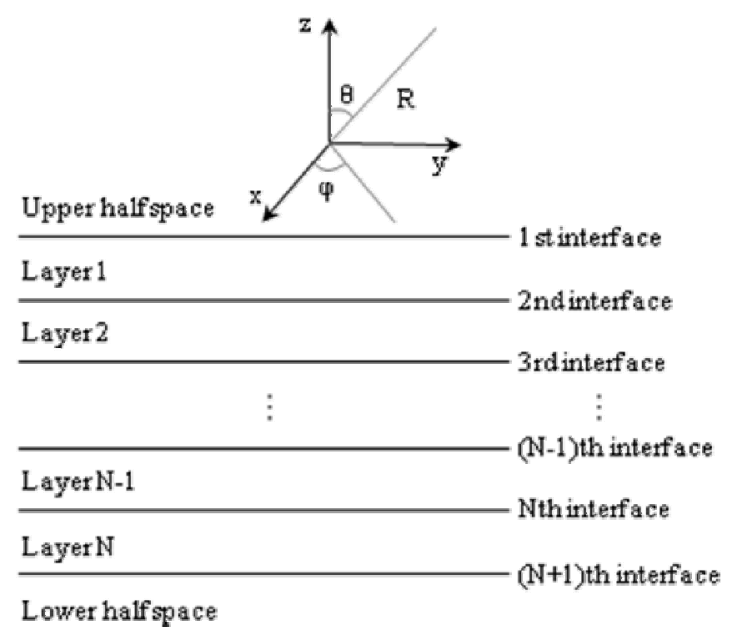

Fig. 1 General planar multi-layer system expressed in the cartesian coordinate system.

If one layer shown in Fig. 1 is an acoustic fluid layer with thickness $h$, pressure in the acoustic fluid layer can be obtained in terms of complex exponential functions as follows:

$$
p(\alpha, \beta, z)=A_{1} \exp (i \gamma z)+A_{2} \exp (-i \gamma z)
$$

where $\gamma=\sqrt{k^{2}-\alpha^{2}-\beta^{2}}$ and $k=\omega / c$ where $c$ is sound speed, $p(\alpha, \beta, z)$ is the Fourier transformed spectral pressure for 
the $x$ and $y$ directions. The displacement in the $\mathrm{z}$ direction and the continuous pressure conditions at the lower surface of a layer, $z=0$ and at the upper surface of a layer, $z=h$ are expressed as follows:

$$
\begin{gathered}
\left(\begin{array}{l}
p(\alpha, \beta, h) \\
p(\alpha, \beta, 0)
\end{array}\right)=\left(\begin{array}{cc}
\exp (i \gamma h) & \exp (-i \gamma h) \\
1 & 1
\end{array}\right)\left(\begin{array}{l}
A_{1} \\
A_{2}
\end{array}\right) \\
\left(\begin{array}{l}
u_{z}(\alpha, \beta, h) \\
u_{z}(\alpha, \beta, 0)
\end{array}\right)=\left(i \gamma / \rho \omega^{2}\right)\left(\begin{array}{cc}
\exp (i \gamma h) & -\exp (-i \gamma h) \\
1 & -1
\end{array}\right)\left(\begin{array}{l}
A_{1} \\
A_{2}
\end{array}\right)
\end{gathered}
$$

Eqs. 2 and 3 can be reduced by eliminating the arbitrary constants $A_{1}$ and $A_{2}$, and the spectral dynamic stiffness matrix equation is giving

$$
[D(\alpha, \beta)]\left(\begin{array}{l}
u_{z}(\alpha, \beta, h) \\
u_{z}(\alpha, \beta, 0)
\end{array}\right)=\left(\begin{array}{l}
S_{z}(\alpha, \beta, h) \\
S_{z}(\alpha, \beta, 0)
\end{array}\right)
$$

Here, the spectral surface traction, $S_{z}(\alpha, \beta, h)=-p(\alpha, \beta, h)$ and $S_{z}(\alpha, \beta, 0)=p(\alpha, \beta, 0)$, are positive when acting in the positive $\mathrm{z}$ direction.

\section{Isotropic elastic layer}

For an isotropic elastic layer with thickness $h$ from Fig. 1, the substitutions for the Cartesian coordinates are

$$
\begin{gathered}
\mathbf{u}=\nabla F+\nabla \times \mathbf{P} \\
\mathbf{P}=(0,0, G)+\nabla \times(0,0,-H)
\end{gathered}
$$

where $\mathbf{u}$ represents the solutions of the time-harmonic displacement equations, in the absence of body forces. Eqs. $5 \sim 6$ reduce the linear displacement equations of elasticity to

$$
\begin{aligned}
& \nabla^{2} F+k_{l}^{2} F=0 \\
& \nabla^{2} G+k_{s}^{2} G=0 \\
& \nabla^{2} H+k_{s}^{2} H=0
\end{aligned}
$$

where $k_{l}=\omega / c_{l}$ and $k_{s}=\omega / c_{s}$ with $c_{l}$ and $c_{s}$ being the wavespeeds of pure longitudinal and shear waves in unbounded media. Using the Fourier transform decompositions from Eqs. 7 9, the general solutions of these equations can be obtained in terms of exponential functions:

$$
\begin{aligned}
& F(\alpha, \beta, z)=A_{1} \exp \left(i \gamma_{l} z\right)+A_{2} \exp \left(-i \gamma_{l} z\right) \\
& G(\alpha, \beta, z)=A_{3} \exp \left(i \gamma_{s} z\right)+A_{4} \exp \left(-i \gamma_{s} z\right) \\
& H(\alpha, \beta, z)=A_{5} \exp \left(i \gamma_{s} z\right)+A_{6} \exp \left(-i \gamma_{s} z\right)
\end{aligned}
$$


where $\gamma_{l}=\sqrt{k_{l}^{2}-\alpha^{2}-\beta^{2}}$ and $\gamma_{s}=\sqrt{k_{s}^{2}-\alpha^{2}-\beta^{2}}$. The spectral displacements of Eq. 5 can be written as

$$
\begin{aligned}
& u_{x}(\alpha, \beta, z)=i \alpha F(\alpha, \beta, z)+i \beta G(\alpha, \beta, z)-i \alpha \frac{\partial H(\alpha, \beta, z)}{\partial z} \\
& u_{y}(\alpha, \beta, z)=i \beta F(\alpha, \beta, z)-i \alpha G(\alpha, \beta, z)-i \beta \frac{\partial H(\alpha, \beta, z)}{\partial z} \\
& u_{z}(\alpha, \beta, z)=\frac{\partial F(\alpha, \beta, z)}{\partial z}-\alpha^{2} H(\alpha, \beta, z)-\beta^{2} H(\alpha, \beta, z)
\end{aligned}
$$

Using Eqs. 13 15, the spectral displacements in terms of the unknown constants $\mathrm{A}_{1}$ to $\mathrm{A}_{6}$ are obtained as

$$
\begin{aligned}
& u_{x}(\alpha, \beta, z)=i \alpha A_{1} \exp \left(i \gamma_{l} z\right)+i \alpha A_{2} \exp \left(-i \gamma_{l} z\right) \\
& +i \beta A_{3} \exp \left(i \gamma_{s} z\right)+i \beta A_{4} \exp \left(-i \gamma_{s} z\right)+\alpha \gamma_{s} A_{5} \exp \left(i \gamma_{s} z\right)-\alpha \gamma_{s} A_{6} \exp \left(-i \gamma_{s} z\right) \\
& u_{y}(\alpha, \beta, z)=i \beta A_{1} \exp \left(i \gamma_{l} z\right)+i \beta A_{2} \exp \left(-i \gamma_{l} z\right) \\
& -i \alpha A_{3} \exp \left(i \gamma_{s} z\right)-i \alpha A_{4} \exp \left(-i \gamma_{s} z\right)+\beta \gamma_{s} A_{5} \exp \left(i \gamma_{s} z\right)-\beta \gamma_{s} A_{6} \exp \left(-i \gamma_{s} z\right) \\
& u_{z}(\alpha, \beta, z)=i \gamma_{l} A_{1} \exp \left(i \gamma_{l} z\right)-i \gamma_{l} A_{2} \exp \left(-i \gamma_{l} z\right) \\
& -\alpha^{2} A_{5} \exp \left(i \gamma_{s} z\right)-\alpha^{2} A_{6} \exp \left(-i \gamma_{s} z\right)-\beta^{2} A_{5} \exp \left(i \gamma_{s} z\right)-\beta^{2} A_{6} \exp \left(-i \gamma_{s} z\right)
\end{aligned}
$$

And by substituting Eqs. 16 18 into the stress-displacement equations, the spectral stresses in terms of the unknown constants are

$$
\begin{aligned}
\tau_{z x}(\alpha, \beta, z)= & -2 \mu \alpha \gamma_{l} A_{1} \exp \left(i \gamma_{l} z\right)+2 \mu \alpha \gamma_{l} A_{2} \exp \left(-i \gamma_{l} z\right)-\mu \beta \gamma_{s} A_{3} \exp \left(i \gamma_{s} z\right)+\mu \beta \gamma_{s} A_{4} \exp \left(-i \gamma_{s} z\right) \\
& +i \mu \alpha\left(k_{s}^{2}-\alpha^{2}-\beta^{2}\right) A_{5} \exp \left(i \gamma_{s} z\right)+i \mu \alpha\left(k_{s}^{2}-\alpha^{2}-\beta^{2}\right) A_{6} \exp \left(-i \gamma_{s} z\right) \\
\tau_{z y}(\alpha, \beta, z)= & -2 \mu \beta \gamma_{l} A_{1} \exp \left(i \gamma_{l} z\right)+2 \mu \beta \gamma_{l} A_{2} \exp \left(-i \gamma_{l} z\right)+\mu \alpha \gamma_{s} A_{3} \exp \left(i \gamma_{s} z\right)-\mu \alpha \gamma_{s} A_{4} \exp \left(-i \gamma_{s} z\right) \\
& +i \mu \beta\left(k_{s}^{2}-\alpha^{2}-\beta^{2}\right) A_{5} \exp \left(i \gamma_{s} z\right)+i \mu \beta\left(k_{s}^{2}-\alpha^{2}-\beta^{2}\right) A_{6} \exp \left(-i \gamma_{s} z\right) \\
\sigma_{z z}(\alpha, \beta, z)= & -\left(\lambda k_{l}^{2}+2 \mu \gamma_{l}^{2}\right) A_{1} \exp \left(i \gamma_{l} z\right)-\left(\lambda k_{l}^{2}+2 \mu \gamma_{l}^{2}\right) A_{2} \exp \left(-i \gamma_{l} z\right) \\
& -2 i \mu \gamma_{s}\left(\alpha^{2}+\beta^{2}\right) A_{5} \exp \left(i \gamma_{s} z\right)+2 i \mu \gamma_{s}\left(\alpha^{2}+\beta^{2}\right) A_{6} \exp \left(-i \gamma_{s} z\right)
\end{aligned}
$$

Similar to the acoustic fluid layer calculations, Eq. 22 can be obtained from Eqs. 16 18 and Eqs. 19 21 by eliminating the unknown constants using the continuous conditions at the lower and upper boundaries of an elastic layer as follows:

$$
[D(\alpha, \beta)]\left(\begin{array}{l}
u_{x}(\alpha, \beta, h) \\
u_{y}(\alpha, \beta, h) \\
u_{z}(\alpha, \beta, h) \\
u_{x}(\alpha, \beta, 0) \\
u_{y}(\alpha, \beta, 0) \\
u_{z}(\alpha, \beta, 0)
\end{array}\right)=\left(\begin{array}{l}
S_{x}(\alpha, \beta, h) \\
S_{y}(\alpha, \beta, h) \\
S_{z}(\alpha, \beta, h) \\
S_{x}(\alpha, \beta, 0) \\
S_{y}(\alpha, \beta, 0) \\
S_{z}(\alpha, \beta, 0)
\end{array}\right)
$$


and the spectral surface tractions,

$$
\left(\begin{array}{l}
S_{x}(\alpha, \beta, h) \\
S_{y}(\alpha, \beta, h) \\
S_{z}(\alpha, \beta, h) \\
S_{x}(\alpha, \beta, 0) \\
S_{y}(\alpha, \beta, 0) \\
S_{z}(\alpha, \beta, 0)
\end{array}\right)=\left(\begin{array}{c}
\tau_{z x}(\alpha, \beta, h) \\
\tau_{z y}(\alpha, \beta, h) \\
\sigma_{z z}(\alpha, \beta, h) \\
-\tau_{z x}(\alpha, \beta, 0) \\
-\tau_{z y}(\alpha, \beta, 0) \\
-\sigma_{z z}(\alpha, \beta, 0)
\end{array}\right)
$$

which are positive when acting in the positive directions of the coordinate axes.

The governing equation of a multi-layer composed of acoustic fluid layers and isotropic elastic layers can be obtained from Eq. 4 for the acoustic fluid layers and Eq. 22 for the isotropic elastic layers as follows:

$$
\mathbf{Z}(\alpha, \beta) \mathbf{u}(\alpha, \beta)=\mathbf{E}(\alpha, \beta)
$$

For a multi-layer structure with $\mathrm{N}$ layers as shown in Fig. $1,(\mathrm{~N}+1)$ boundary surfaces exist at the model. The spectral stiffness matrix $\mathbf{Z}(\alpha, \beta)$ is a $3(\mathrm{~N}+1) \times 3(\mathrm{~N}+1)$ matrix, and the spectral displacements vector $\mathbf{u}(\alpha, \beta)$ and the spectral excitation vector $\mathbf{E}(\alpha, \beta)$ are $3(\mathrm{~N}+1) \times 1$ column vectors.

\section{EXTERNAL EXCITATIONS}

\section{Mechanical excitation}

The main excitations that influence the HMS of a submerged vehicle during military activity can be classified into three types: mechanical excitation, plane wave excitation, and turbulent flow excitation. Mechanical excitation is induced when a submerged vehicle's essential equipment is operated. The noise due to the mechanical excitation is transmitted to sensors of the HMS through a submerged vehicle pressure hull.

When mechanical excitation is applied to the multi-layer system, the spectral excitation vector $\mathbf{E}(\alpha, \beta)$ is zero excluding the degrees of freedom where the excitation is acting. If the point force vector, $\mathbf{F}=\left(F_{x}, F_{y}, F_{z}\right)$ acts at $x=x_{0}, y=y_{0}$ of the jth interface of Fig. 1, then all elements except for the elements

$$
\begin{aligned}
& E_{3 j-2}=F_{x} \exp \left(-i \alpha x_{0}-i \beta y_{0}\right) \\
& E_{3 j-1}=F_{y} \exp \left(-i \alpha x_{0}-i \beta y_{0}\right) \\
& E_{3 j}=F_{z} \exp \left(-i \alpha x_{0}-i \beta y_{0}\right)
\end{aligned}
$$

are zero. With the spectral displacements of each layer obtained from Eq. 24, the far field pressure in the upper halfspace at observation angles $(\theta, \varphi)$ can be obtained as follows:

$$
p_{f f}(R, \theta, \varphi)=-\rho_{u} \omega^{2} U_{u}\left(\alpha_{0}, \beta_{0}\right) \frac{\exp \left(i k_{u} R\right)}{2 \pi R}
$$

where the subscript ' $\mathrm{u}$ ' signifies the upper acoustic domain in which the pressure propagates. $U_{u}\left(\alpha_{0}, \beta_{0}\right)$ is a z directional spectral displacement of a boundary surface between multi-layer system and the upper acoustic domain, the $1^{\text {st }}$ interface of Fig. 1 . 
$\alpha_{0}=k_{u} \sin \theta \cos \varphi$ and $\beta_{0}=k_{u} \sin \theta \sin \varphi$ are the stationary phase wavenumbers.

\section{Multi-layer including stiffened layer}

Mace (1980) developed a sound radiation from a stiffener reinforced thin plate. He expressed the transverse displacement of the neutral axis of the thin plate which was reinforced by parallel stiffeners with a spacing $d$ by Fourier transform in $\mathrm{x}$ and $\mathrm{y}$ coordinates as follows:

$$
W(\alpha, \beta)=\frac{S_{z}(\alpha, \beta)}{D_{s}(\alpha, \beta)}-\frac{G_{s}(\beta) \sum_{n=-\infty}^{\infty} \frac{S_{z}(\alpha-2 \pi n / d, \beta)}{D_{s}(\alpha-2 \pi n / d, \beta)}}{D_{s}(\alpha, \beta)\left(1+G_{s}(\beta) \sum_{n=-\infty}^{\infty} \frac{1}{D_{s}(\alpha-2 \pi n / d, \beta)}\right)}
$$

where $G_{s}(\beta)=\left(E_{s} I_{s} \beta^{4}-\omega^{2} \rho_{s} A_{s}\right) / d . E_{s}$ is Young's modulus of the stiffener, $I_{s}$ is the cross-section area moment of inertia, $\rho_{s}$ is density and $A_{s}$ is the cross-section area.

This equation can be rearranged to the form of the stiffness of the plate $D_{s}(\alpha, \beta)$, the transverse displacement $W(\alpha, \beta)$, and the force acting on the plate as follows:

$$
D_{s}(\alpha, \beta) W(\alpha, \beta)=S_{z}(\alpha, \beta)-\frac{G_{s}(\beta) \sum_{n=-\infty}^{\infty} \frac{S_{z}(\alpha-2 \pi n / d, \beta)}{D_{s}(\alpha-2 \pi n / d, \beta)}}{1+G_{s}(\beta) \sum_{n=-\infty}^{\infty} \frac{1}{D_{s}(\alpha-2 \pi n / d, \beta)}}
$$

The first term on the right-hand side of the Eq. 30 is the external mechanical force acting on the thin plate, and the second term is the extra force due to stiffeners in the transverse direction. Here, these forces are acting on the bottom surface of the plate. If the stiffeners are attached to the jth interface of the multi-layer and the external force acts at the same surface, then, the excitation element, Eq. 27 for a stiffened multi-layer system can be obtained as:

$$
E_{3 j}=F_{z} \exp \left(-i \alpha x_{0}-i \beta y_{0}\right)-\frac{G_{s}(\beta) \sum_{n=-\infty}^{\infty} \frac{S_{z}(\alpha-2 \pi n / d, \beta)}{D_{s}(\alpha-2 \pi n / d, \beta)}}{1+G_{s}(\beta) \sum_{n=-\infty}^{\infty} \frac{1}{D_{s}(\alpha-2 \pi n / d, \beta)}}
$$

With Eq. 31, the stiffened multi-layer structure can be evaluated for the mechanical excitation.

\section{Plane wave excitation}

Plane wave excitation occurs when a signal from other ships or submerged vehicles reaches the HMS system of a submerged vehicle. The signal can be assumed as a plane wave because sources of the signal are usually located far from the submerged vehicle. Thus, exterior noise can be classified as plane wave excitation.

When an incident plane wave comes from the upper halfspace to the multi-layer structure at angles $\left(\theta_{i}, \varphi_{i}\right)$, the incident wave pressure is

$$
p_{i}(x, y, z)=A \exp \left(-i \alpha_{i} x-i \beta_{i} y-i \gamma_{i} z\right)
$$

where $\alpha_{i}=k_{u} \sin \theta_{i} \cos \varphi_{i}, \beta_{i}=k_{u} \sin \theta_{i} \sin \varphi_{i}, \gamma_{i}=k_{u} \cos \theta_{i}$, and $k_{u}=\omega / c_{u}$ where $c_{u}$ is sound speed at the upper halfspace and the subscript ' $u$ ' signifies the acoustic domain of the incident wave. 
The plane wave affects the first surface of the layered system, and every element of the spectral excitation vector $\mathbf{E}(\alpha, \beta)$ is zero except for the element

$$
E_{3}=-2 A
$$

The spectral displacements can be calculated using Eq. 24 and Eq. 33. With those displacements, the complex reflection coefficient is obtained as follows:

$$
R=1+\frac{-\rho_{u} \omega^{2} U_{u}\left(-\alpha_{i},-\beta_{i}\right)}{i A k_{u} \cos \theta_{i}}
$$

where $\rho_{u}$ is the density of the upper halfspace. $U_{u}\left(-\alpha_{i},-\beta_{i}\right)$ is the $\mathrm{z}$ directional displacement of the upper boundary of the top layer.

\section{Turbulent flow excitation}

Submerged vehicle movement causes turbulent flow excitation. When the submerged vehicle carries out a military operation, turbulent flows are generated on the exterior surface of the vessel. Theses flows produce noise which affects the HMS system.

Ko and Schloemer (1989) Ko and Nuttall (1991) presented a method for evaluating noise caused by turbulent flow using the turbulent boundary layer pressure model. He defined a turbulent flow induced noise that affected a point hydrophone exposed to the water field as the frequency spectral density using the Corcos (1964) tur-bulent wall pressure spectra which is written as

$$
Q(\omega)=2 \pi \iint_{-\infty}^{\infty} P\left(k_{x}, k_{y}, \omega\right) d k_{x} d k_{y}
$$

where $P\left(k_{x}, k_{y}, \omega\right)$ is the turbulent wall pressure spectrum.

If the point hydrophone is embedded within a certain layer, the frequency spectral density is

$$
Q(\omega)=2 \pi \int_{-\infty}^{+\infty} \int_{-\infty}^{+\infty} P\left(k_{x}, k_{y}, \omega\right) T\left(k_{x}, k_{y}, \omega\right) d k_{x} d k_{y}
$$

where $T\left(k_{x}, k_{y}, \omega\right)$ is the transfer function which is the ratio of the normal stress of the point hydrophone to the force acting on the flow contact surface of layered system, and defined as

$$
T\left(k_{x}, k_{y}, \omega\right)=\left|\frac{T_{z z}\left(k_{x}, k_{y}, \omega, z\right)_{z=\text { sensor }}}{F_{0}\left(k_{x}, k_{y}, \omega\right)_{z=\text { surface }}}\right|^{2}
$$

Ko and Schloemer (1989) Ko, Pyo and Seong (2001) found $T_{z z}$ case by case for two- and three-layer models possessing an embedded hydrophone in the elastomer layer. However, $T_{z z}$ can be replaced by Eq. 1 for an acoustic fluid layer and Eq. 21 for an isotropic elastic layer. With this relation, turbulent flow analysis method can be applied to general multi-layer models. 


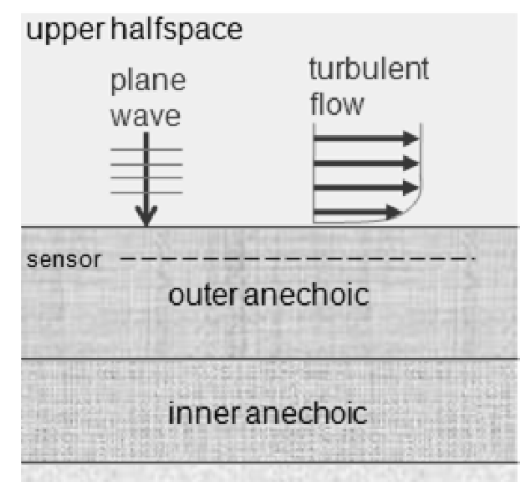

buoyancy material

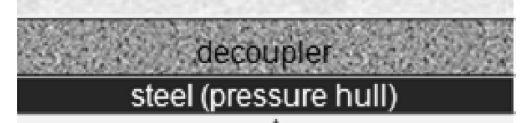

lowerhalfspace $\uparrow$

$$
\begin{aligned}
& \text { mechanical } \\
& \text { force }
\end{aligned}
$$

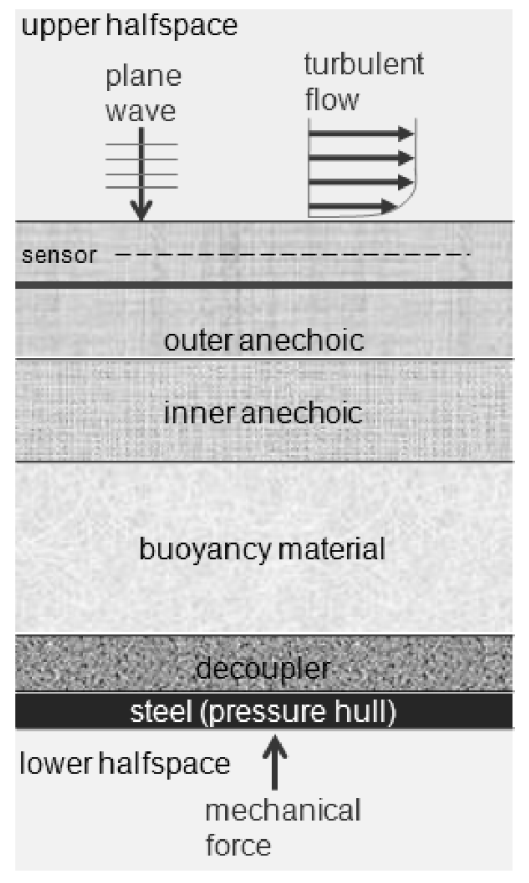

(b)

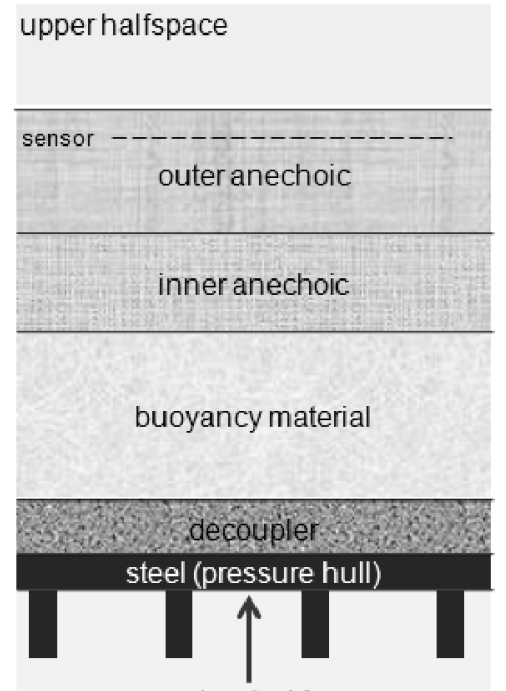

lowerhalfspace

(c)

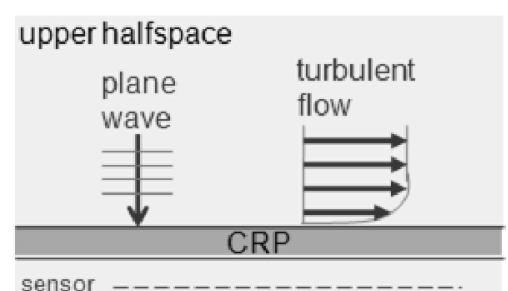

sea water

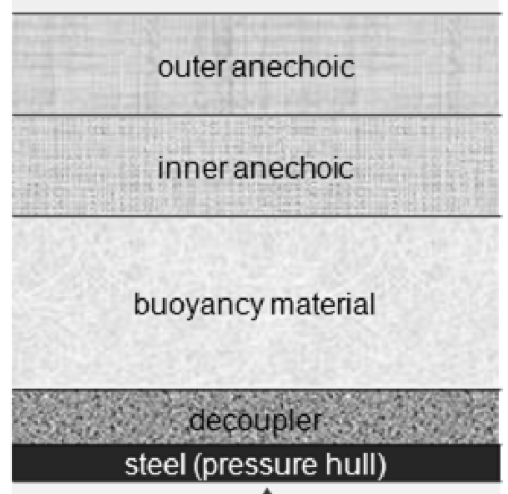

lowerhalfspace $\uparrow$

mechanical
force

(d)

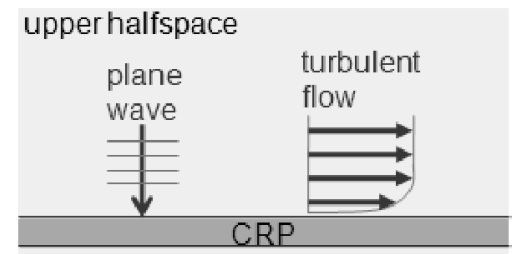

sea water

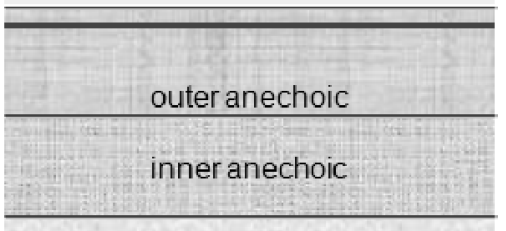

buoyancy material

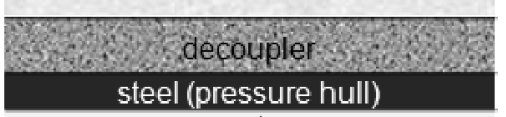

lowerhalfspace $\uparrow$

mechanical force

(e)

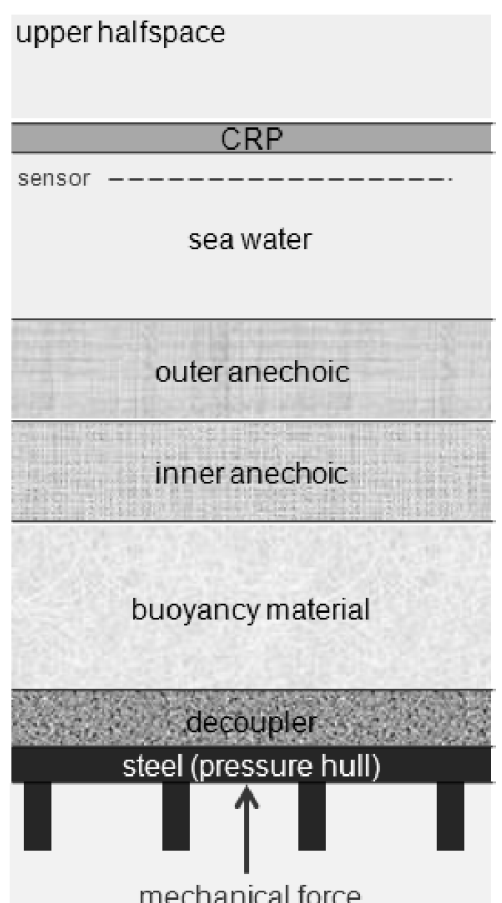

lower halfspace

(f)

Fig. 2 Planar multi-layer models. (a) Sensor in the outer anechoic layer; (b) aluminum layer added in the outer anechoic layer of model (a); (c) stiffeners reinforced in the model (a);

(d) sensor in the sea water layer; (e) aluminum layer added in the outer anechoic layer of model (d); (f) stiffeners reinforced in the model (d). 
Table 1 Material properties of each layer in Fig. 2a.

\begin{tabular}{|c|c|c|c|c|c|c|}
\hline Layer no. & Material & $\mathrm{E}\left[N / m^{2}\right]$ & $\sigma$ & $\rho\left[\mathrm{kg} / \mathrm{m}^{3}\right]$ & $\eta$ & $\mathrm{h}[m]$ \\
\hline upper & S. W. & & & 1500 & & \\
\hline 1 & O. A. & $6.4 \mathrm{e} 7$ & 0.48 & 1600 & 0.54 & 0.090 \\
\hline 2 & I. A. & $3.9 \mathrm{e} 7$ & 0.48 & 1500 & 0.52 & 0.070 \\
\hline 3 & B. M. & $4.5 \mathrm{e} 8$ & 0.43 & 300 & 0.01 & 0.120 \\
\hline 4 & decoupler & $2.6 \mathrm{e} 7$ & 0.46 & 800 & 0.47 & 0.040 \\
\hline 5 & steel & $2.1 \mathrm{e} 11$ & 0.3 & 7800 & 0.001 & 0.025 \\
\hline lower & S. W. / air & & & $1500 / 343$ & & \\
\hline
\end{tabular}

Table 2 Material properties of each layer in Fig. $2 b$.

\begin{tabular}{|c|c|c|c|c|c|c|}
\hline Layer no. & Material & $\mathrm{E}\left[N / m^{2}\right]$ & $\sigma$ & $\rho\left[\mathrm{kg} / \mathrm{m}^{3}\right]$ & $\eta$ & $\mathrm{h}[m]$ \\
\hline upper & S. W. & & & 1500 & & \\
\hline 1 & O. A. & $6.4 \mathrm{e} 7$ & 0.48 & 1600 & 0.54 & 0.040 \\
\hline 2 & Aluminum & $7.0 \mathrm{e} 10$ & 0.33 & 2700 & 0.001 & 0.005 \\
\hline 3 & O. A. & $6.4 \mathrm{e} 7$ & 0.48 & 1600 & 0.54 & 0.050 \\
\hline 4 & I. A. & $3.9 \mathrm{e} 7$ & 0.48 & 1500 & 0.52 & 0.070 \\
\hline 5 & B. M. & $4.5 \mathrm{e} 8$ & 0.43 & 300 & 0.01 & 0.120 \\
\hline 6 & decoupler & $2.6 \mathrm{e} 7$ & 0.46 & 800 & 0.47 & 0.040 \\
\hline 7 & steel & $2.1 \mathrm{e} 11$ & 0.3 & 7800 & 0.001 & 0.025 \\
\hline lower & S. W. / air & & & $1500 / 343$ & & \\
\hline
\end{tabular}

Noise reduction is defined as the ratio of Eq. 35 to Eq. 36 for the point hydrophone embedded multi-layer system:

$$
N R=10 \log _{10} \frac{[Q(\omega)]_{\text {sensor }}}{[Q(\omega)]_{\text {surface }}}
$$

Noise reduction predicts the sensitivity of the location of the hydrophone or a material property of the hydrophone embedded layer.

\section{RESULT}

The HMS can be classified into two categories defending on the sensor position. In the first category, the sensor is embedded within the elastomer layer. Secondly, the sensor is exposed to water, and a sonar dorm detects the sensor. These two types are simplified in Figs. 2a and 2d, respectively. Models that possess an additional aluminum layer within the outer anechoic layer were considered (Figs. $2 b$ and 2e). The aluminum layer enhances a sensor's ability to receive signals. The HMS may be installed on a surface over a water ballast tank. Thus, models were evaluated to account for both cases in which the HMS was installed in the air and in a water filled area at the inner space of the pressure hull. The sensor was assumed to be $0.02 \mathrm{~m}$ from the upper surface of the layer that contained the sensor; the outer anechoic layer for Figs. $2 \mathrm{a} \sim 2 \mathrm{c}$, and the sea 
Table 3 Material properties of each layer in Fig. $2 \mathrm{~d}$.

\begin{tabular}{|c|c|c|c|c|c|c|}
\hline Layer no. & Material & $\mathrm{E}\left[N / m^{2}\right]$ & $\sigma$ & $\rho\left[\mathrm{kg} / \mathrm{m}^{3}\right]$ & $\eta$ & $\mathrm{h}[m]$ \\
\hline upper & S. W. & & & 1500 & & \\
\hline 1 & CRP & $3.7 \mathrm{e} 10$ & 0.17 & 1500 & 0.02 & 0.020 \\
\hline 2 & S. W. & & & 1500 & & 0.120 \\
\hline 3 & O. A. & $6.4 \mathrm{e} 7$ & 0.48 & 1600 & 0.54 & 0.070 \\
\hline 4 & I. A. & $3.9 \mathrm{e} 7$ & 0.48 & 1500 & 0.52 & 0.070 \\
\hline 5 & B. M. & $4.5 \mathrm{e} 8$ & 0.43 & 300 & 0.01 & 0.120 \\
\hline 6 & decoupler & $2.6 \mathrm{e} 7$ & 0.46 & 800 & 0.47 & 0.040 \\
\hline 7 & steel & $2.1 \mathrm{e} 11$ & 0.3 & 7800 & 0.001 & 0.025 \\
\hline lower & S. W. / air & & & $1500 / 343$ & & \\
\hline
\end{tabular}

Table 4 Material properties of each layer in Fig. 2e.

\begin{tabular}{|c|c|c|c|c|c|c|}
\hline Layer no. & Material & $\mathrm{E}\left[N / m^{2}\right]$ & $\sigma$ & $\rho\left[\mathrm{kg} / \mathrm{m}^{3}\right]$ & $\eta$ & $\mathrm{h}[m]$ \\
\hline upper & S. W. & & & 1500 & & \\
\hline 1 & CRP & $3.7 \mathrm{e} 10$ & 0.17 & 1500 & 0.02 & 0.020 \\
\hline 2 & S. W. & & & 1500 & & 0.120 \\
\hline 3 & O. A. & $6.4 \mathrm{e} 7$ & 0.48 & 1600 & 0.54 & 0.010 \\
\hline 4 & Aluminum & $7.0 \mathrm{e} 10$ & 0.33 & 2700 & 0.001 & 0.005 \\
\hline 5 & O. A. & $6.4 \mathrm{e} 7$ & 0.48 & 1600 & 0.54 & 0.060 \\
\hline 6 & I. A. & $3.9 \mathrm{e} 7$ & 0.48 & 1500 & 0.52 & 0.070 \\
\hline 7 & B. M. & $4.5 \mathrm{e} 8$ & 0.43 & 300 & 0.01 & 0.120 \\
\hline 8 & decoupler & $2.6 \mathrm{e} 7$ & 0.46 & 800 & 0.47 & 0.040 \\
\hline 9 & steel & $2.1 \mathrm{e} 11$ & 0.3 & 7800 & 0.001 & 0.025 \\
\hline lower & S. W. / air & & & $1500 / 343$ & & \\
\hline
\end{tabular}

water layer for Figs. $2 \mathrm{~d} \sim 2 \mathrm{f}$.

Sensitivity of layers thickness for the model Fig. 2a is shown in Fig. 3. The difference between the maximum pressure level and the minimum pressure level increased as the analysis frequency increased. The thickness of the decoupler layer adjacent to the pressure hull was the most sensitive for all frequency ranges.

Fig. 4 shows the pressure level at the sensor position when the mechanical excitation is acting at the bottom of the pressure hull. The pressure levels of the sensor embedded cases were smaller than the sensor in the fluid layer at high frequency. The aluminum layer did not significantly influence the result for mechanical excitation. The pressure level in the low frequency range was smaller when the water was filled the lower halfspace.

Stiffened multi-layer models excited by mechanical force are compared in Fig. 5. Stiffeners were $2 \mathrm{~mm}$ thick and $5 \mathrm{~mm}$ long, and attached to the bottom of the pressure hull with $0.02 \mathrm{~m}$ between stiffeners (Figs. $2 \mathrm{c}$ and $2 \mathrm{f}$ ). The pressure level decreased at the high frequency range for the stiffened models. When the lower halfspace was filled with the air, the stiffener performed better.

Fig. 6 illustrates the pressure level at the sensor position due to the normally incident plane wave from the upper halfspace. The pressure levels of the sensor embedded cases were smaller than the sensor in the fluid layer over all analysis frequency range. The aluminum layer enhanced the pressure level for the sensor embedded model, but the pressure level decreased near the aluminum layer at high frequency when the sensor was located in the fluid layer. 

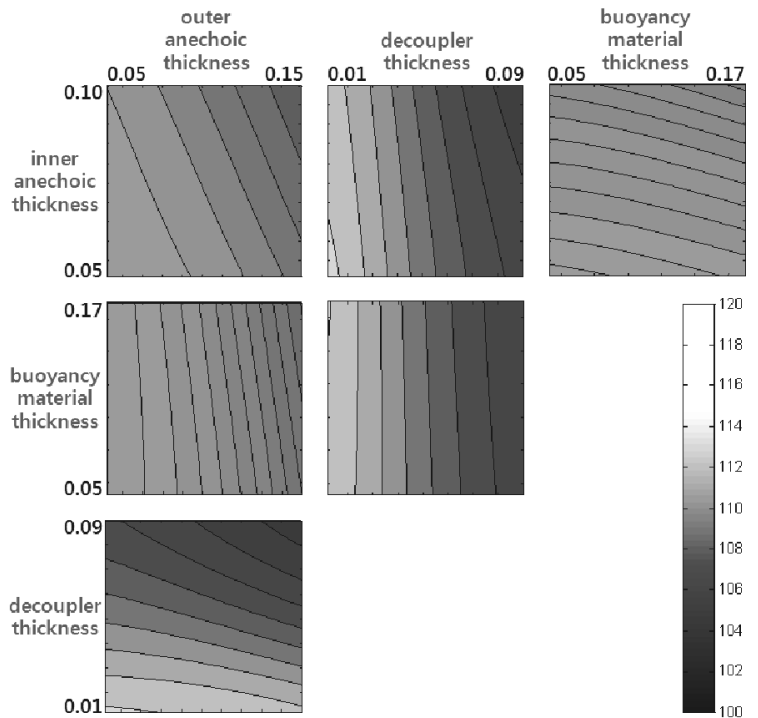

(a) $1 \mathrm{kHz}$.
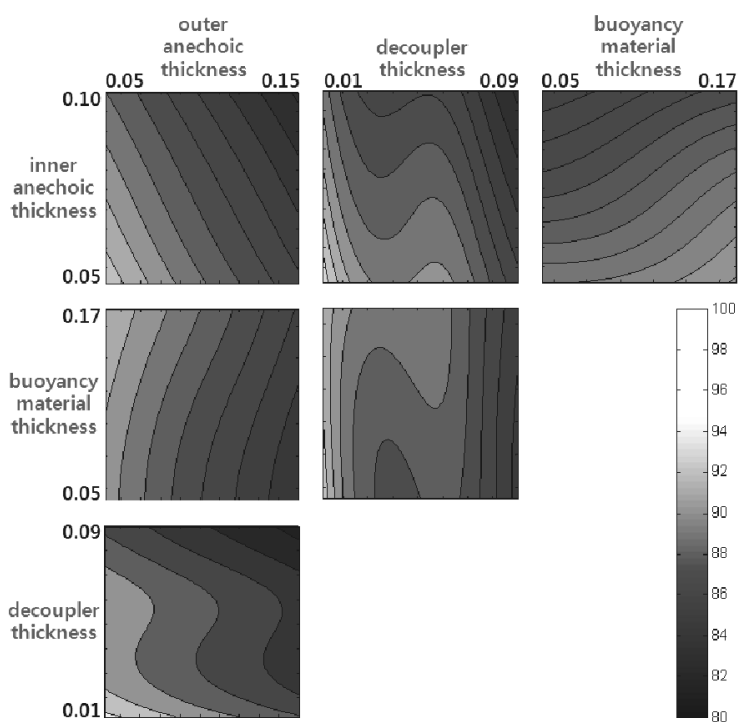

(c) $3 \mathrm{kHz}$.

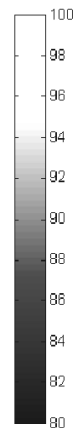

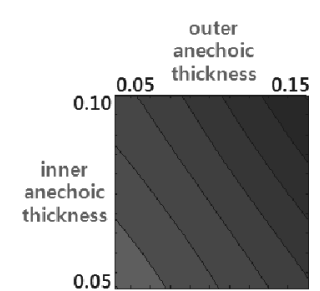

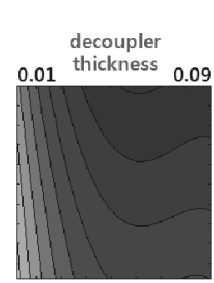

buoyancy

thickness
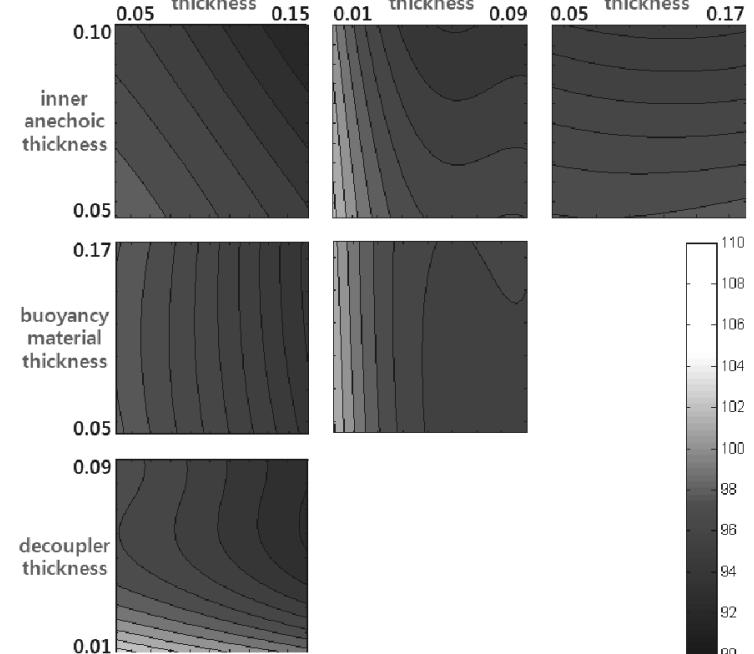

(b) $2 \mathrm{kHz}$.
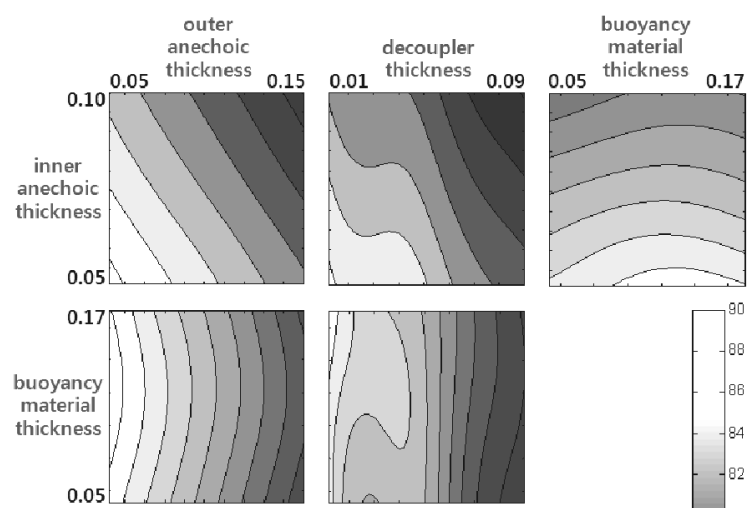

Fig. 3 Acoustic characteristic sensitivity of sound-absorbing layer thickness.

Pressure is in $\mathrm{dB}$ re 1 micropascal. Unit of thickness is a meter.

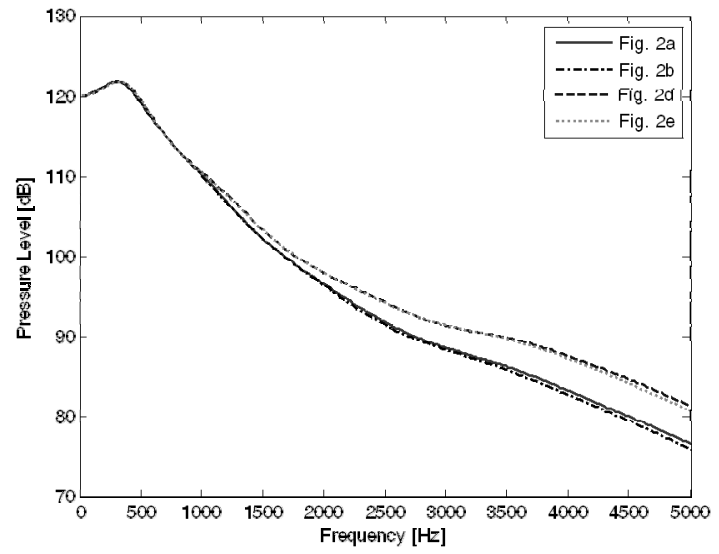

(a) Air filled in the lower halfspace.

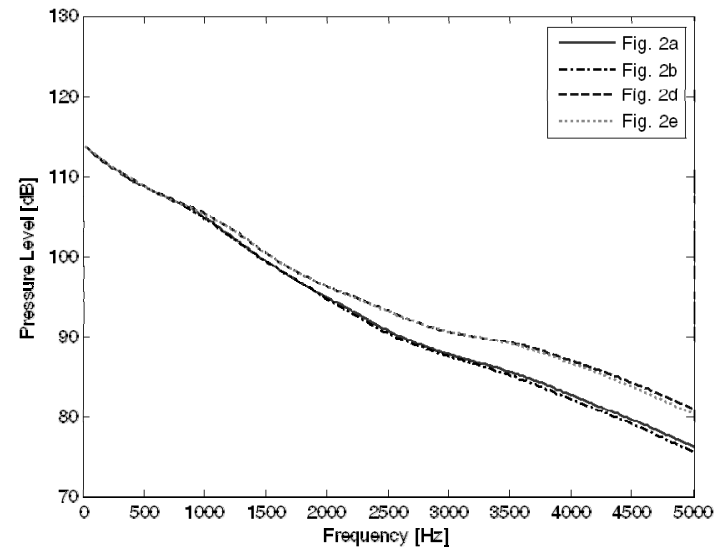

(b) Water filled in the lower halfspace.

Fig. 4 Pressure levels excited by mechanical forces when calculated point is in the sensor position. Stationary phase is $\theta=0^{\circ}$ and $\varphi=0^{\circ}$. 


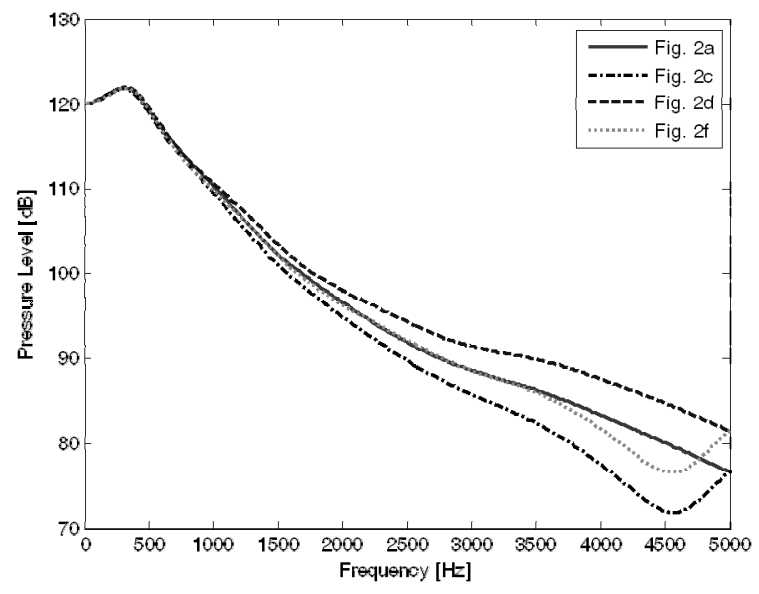

(a) Air filled in the lower halfspace.

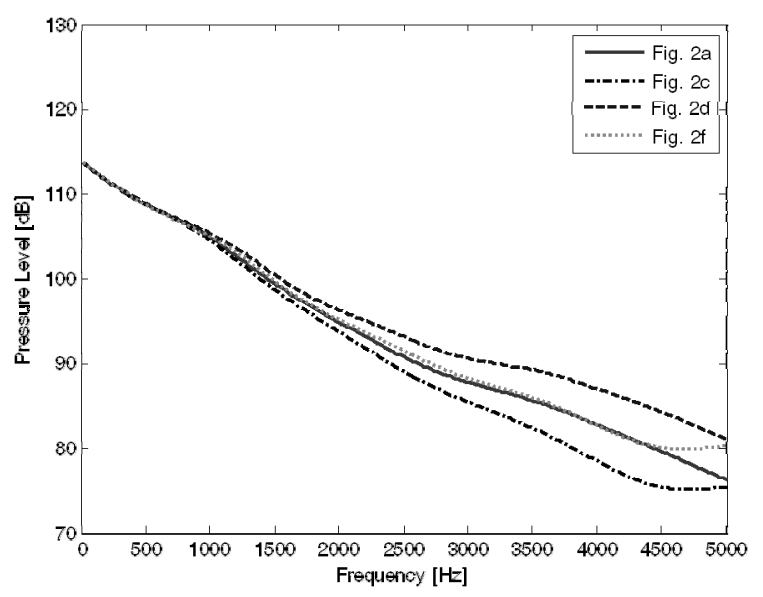

(b) Water filled in the lower halfspace.

Fig. 5 Pressure levels excited by mechanical forces when calculated point is in the sensor position. Stationary phase is $\theta=0^{\circ}$ and $\varphi=0^{\circ}$.

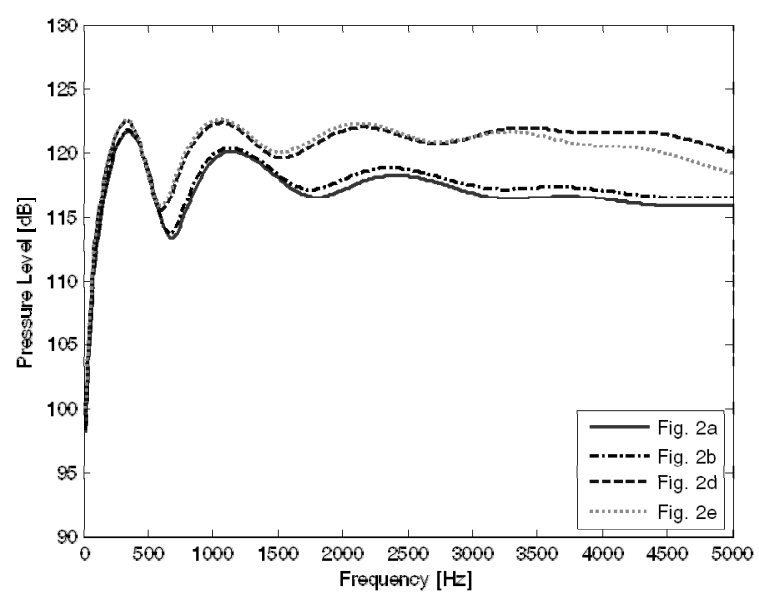

(a) Air filled in the lower halfspace.

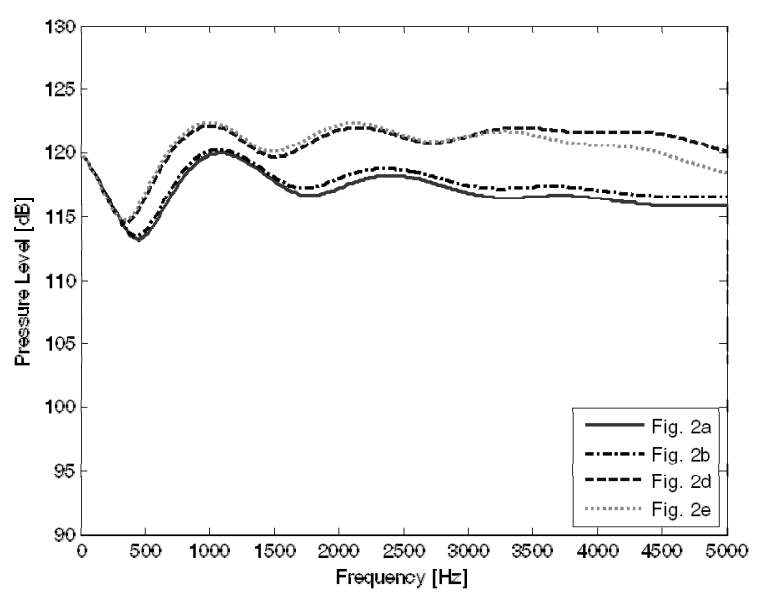

(b) Water filled in the lower halfspace.

Fig. 6 Pressure level excited by plane waves when the calculated point is in the sensor position. Incident angle is $\theta=0^{\circ}$ and $\varphi=0^{\circ}$.

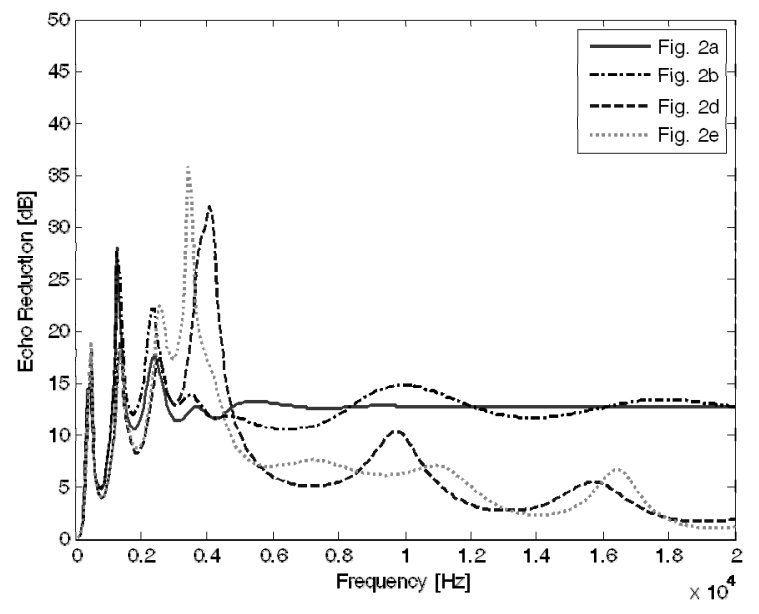

(a) Air filled in the lower halfspace.

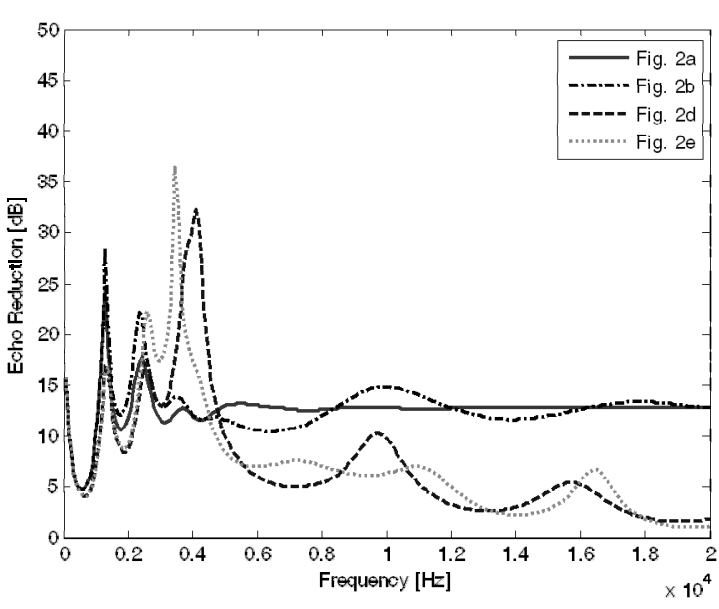

(b) Water filled in the lower halfspace.

Fig. 7 Echo reduction excited by plane waves when the calculated point is in the sensor position. Incident angle is $\theta=0^{\circ}$ and $\varphi=0^{\circ}$. 


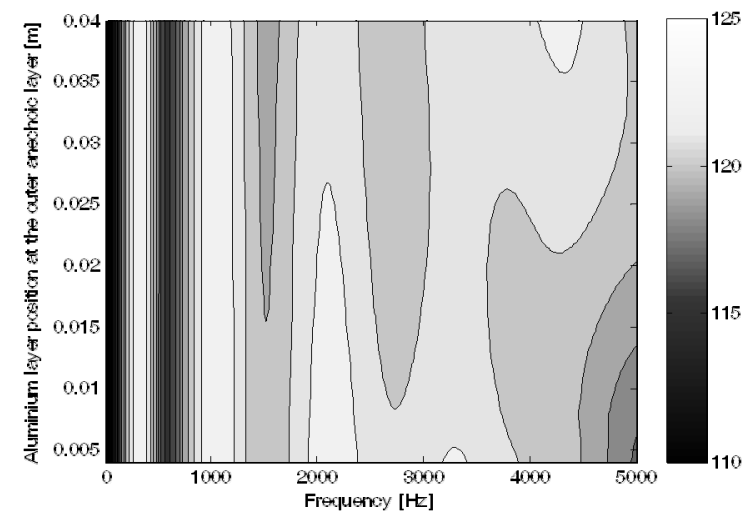

(a) Air filled in the lower halfspace.

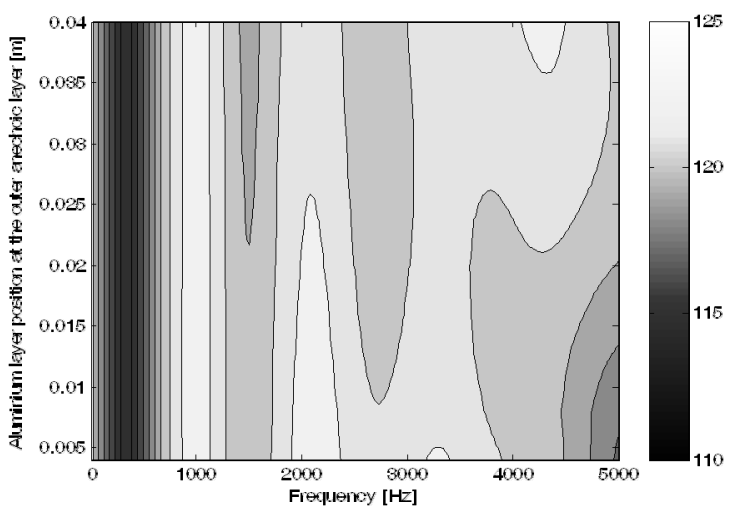

(b) Water filled in the lower halfspace.

Fig. 8 Pressure level excited by plane wave when the location of the aluminum layer is changed and when the calculated point is in the sensor position. Incident angle is $\theta=0^{\circ}$ and $\varphi=0^{\circ}$.

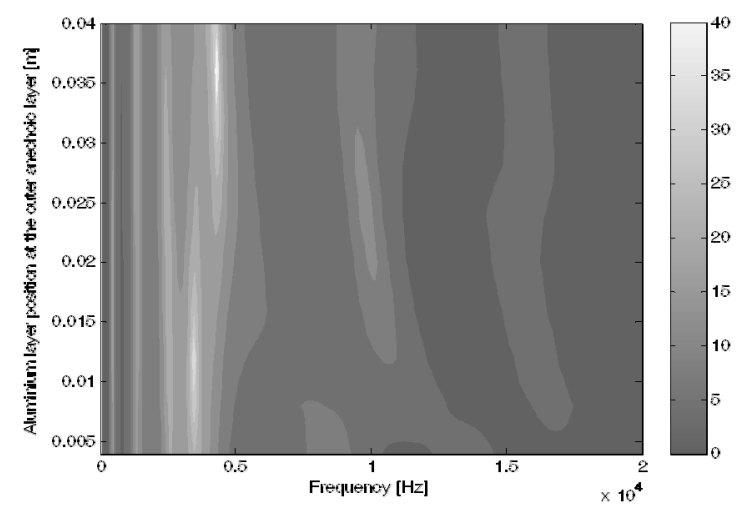

(a) Air filled in the lower halfspace.

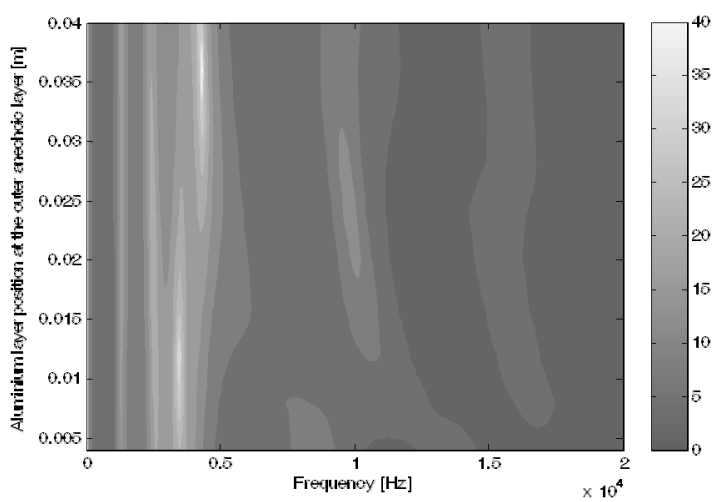

(b) Water filled in the lower halfspace.

Fig. 9 Echo reduction excited by plane waves when the aluminum layer location is changed and when the calculated point is in the sensor position. Incident angle is $\theta=0^{\circ}$ and $\varphi=0^{\circ}$.

Fig. 7 shows the echo reduction of multi-layer models. The position and thickness of the aluminum layer were varied in order to observe the aluminum layer's effects on pressure level and echo reduction. The analysis was investigated for the model Fig. 2 d. The results caused by the position variation are shown in Figs. 8 and 9. The results caused by the thickness variation are shown in Figs. 10 and 11.

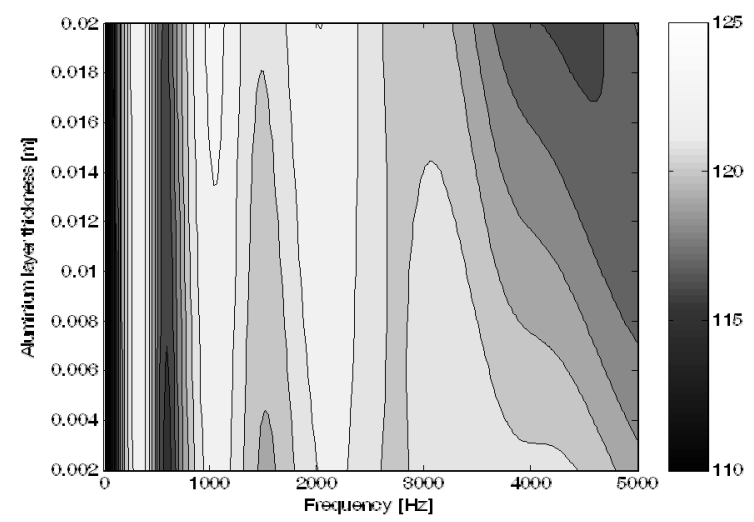

(a) Air filled in the lower halfspace.

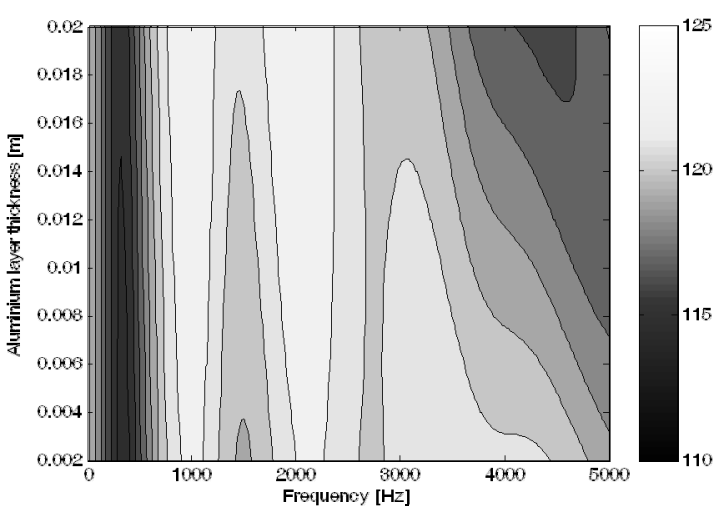

(b) Water filled in the lower halfspace.

Fig. 10 Pressure level excited by plane wave when the aluminum layer thickness is varied and when the calculated point is in the sensor position. Incident angle is $\theta=0^{\circ}$ and $\varphi=0^{\circ}$. 


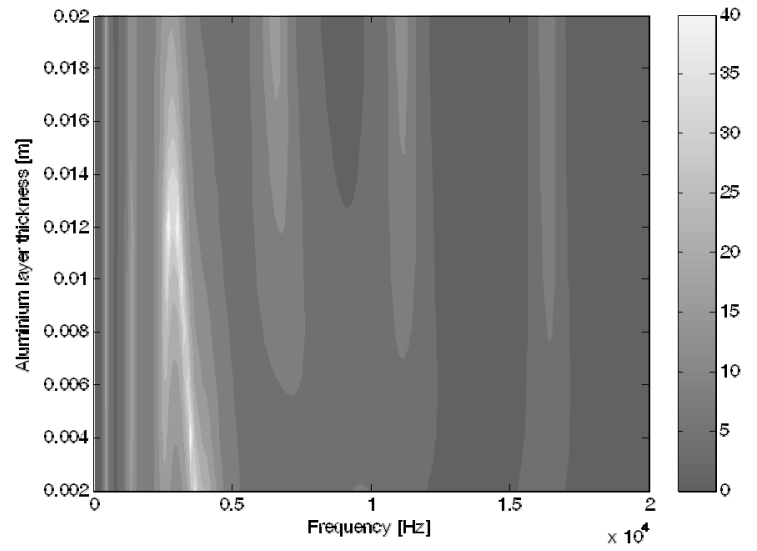

(a) Air filled in the lower halfspace.

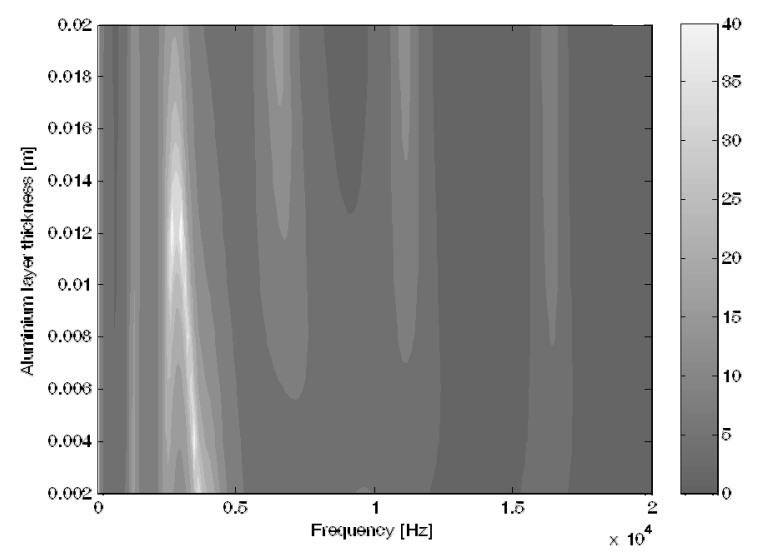

(b) Water filled in the lower halfspace.

Fig. 11 Echo reduction excited by plane waves when the aluminum layer thickness is varied and when the calculated point is in the sensor position. Incident angle is $\theta=0^{\circ}$ and $\varphi=0^{\circ}$.

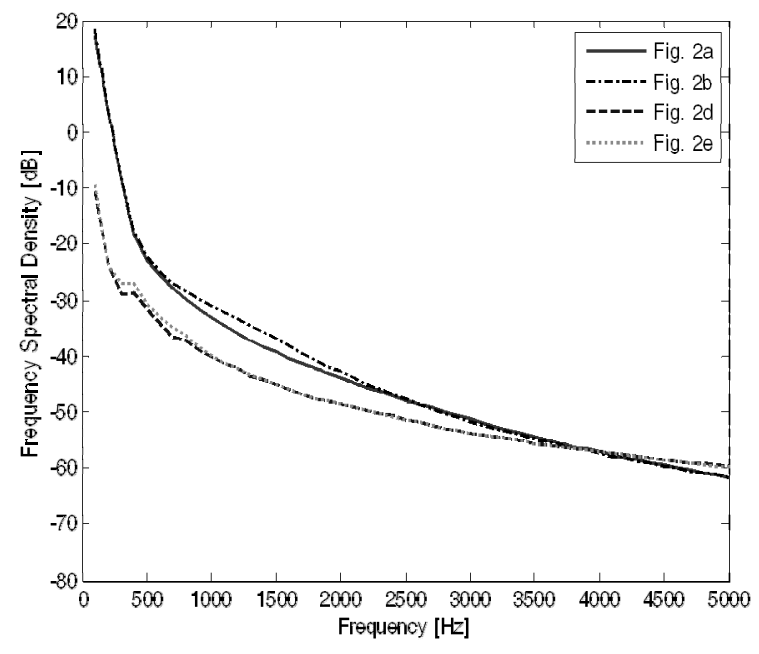

(a) Frequency spectral density.

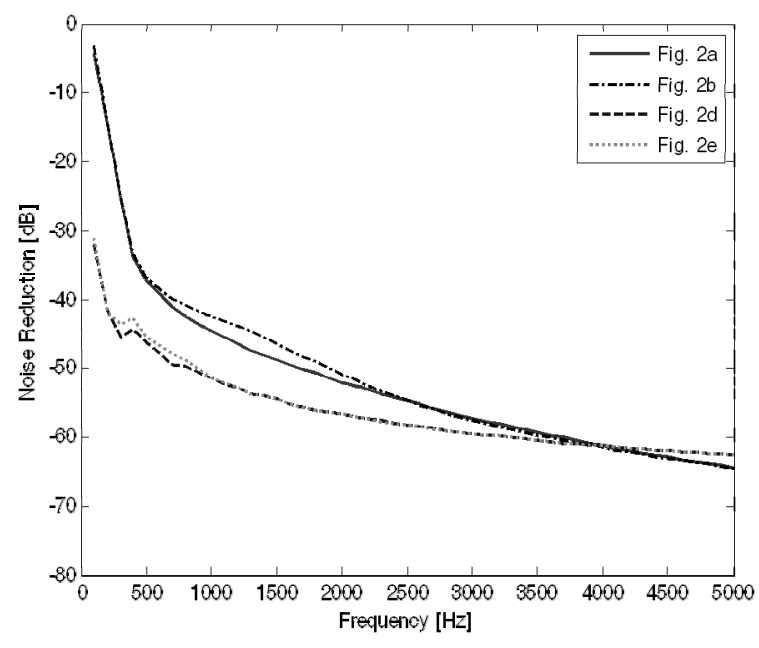

(b) Noise reduction.

Fig. 12 Comparisons for various multi-layer models filled with air in the lower halfspace at the sensor position excited by turbulent flow. Free stream velocity is $10 \mathrm{~m} / \mathrm{s}$.

Pressure level and the echo reduction decreased as the aluminum layer embedded deeper from the upper surface of the sea water layer. However, the pressure level increased in the high frequency range. The pressure increase was especially observed in models filled with water in the lower halfspace. Fig. 12 illustrates the frequency spectral density and the noise reduction at the sensor position for various turbulent wall pressure spectrums.

\section{DISCUSSION}

The various HMS multi-layer systems excited by three external force were evaluated using the infinite planar theory. A good HMS multi-layer will reduce the pressure level at the sensor when a mechanical force or a turbulent flow acts on the HMS. In addition, when an plane wave comes far from the outside, the good HMS will guarantee regular and high signals for the frequency range fewer than $4 \mathrm{kHz}$, and will reduce reflected signals over $4 \mathrm{kHz}$ frequency range. Thus, Fig. $2 \mathrm{~d}$ represents a satisfactory HMS multi-layer system of the analyzed models. If the model Fig. 2a is applied to an actual HMS system, it is recommended that the aluminum layer be added. The HMS evaluation method can account for thickness variations as well as 


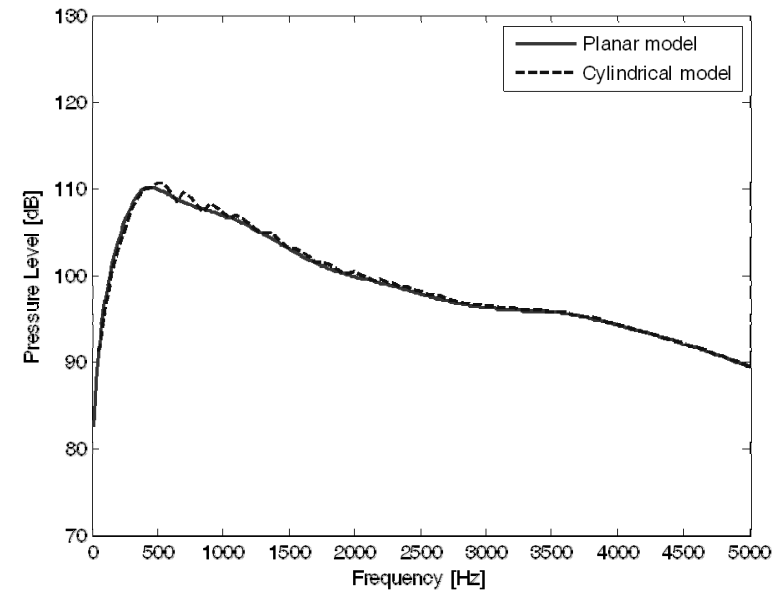

(a) Far-field pressure level excited by mechanical force when calculation point is $\mathrm{R}=1, \theta=0^{\circ}$, and $\varphi=0^{\circ}$ from the surface.

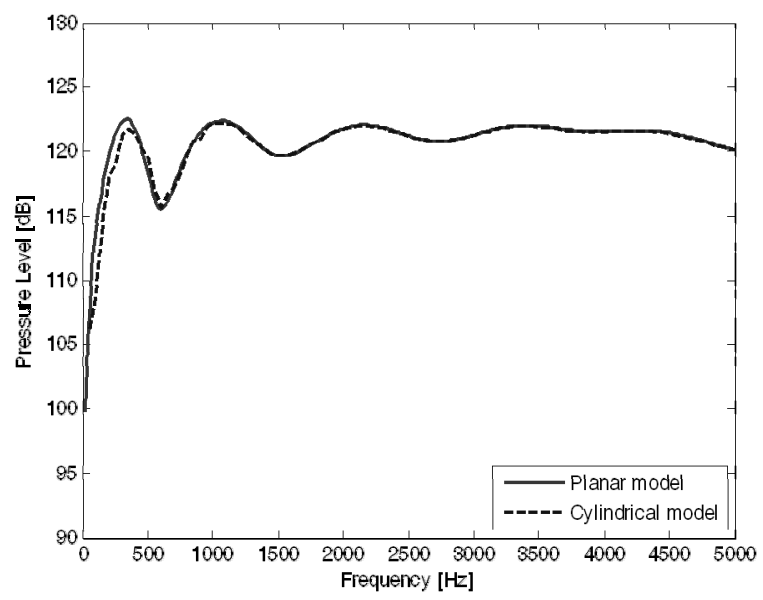

(b) Pressure level excited by plane wave when calculated point is sensor position (Incident angle is $\theta=0^{\circ}$ and $\varphi=0^{\circ}$ ).

Fig. 13 Pressure level comparison between the planar model and the cylindrical model for Fig. 2d filled with air in the lower halfspace in $\mathrm{dB}$ re 1 micropascal.

variations in layer order or layer material. The material property depending on the temperature or the hydraulic pressure can be also investigated with these processors. Fig. $2 \mathrm{c}$ and $2 \mathrm{f}$ illustrate that stiffeners have an effect on the acoustic performance at certain frequency ranges which is depend on the geometry of stiffeners. Stiffened multi-layers have lower pressure levels in Fig 5.

The geometry of a submerged vehicle is similar to a cylinder; thus, an infinite cylindrical multi-layer theory was compared for the model Fig. 2d in Fig. 13. The far-field pressure caused by the mechanical forces is expressed as the summation form of the Bessel functions:

$$
p_{e f}(R, \theta, \phi) \cong \frac{-i \rho \omega^{2}}{\pi k \sin \theta} \frac{\exp (i k R)}{R} \sum_{n=-\infty}^{\infty} \frac{(-i)^{|n|} u_{1}\left(n, \alpha_{0}\right)}{H_{|n|}^{\prime}(k a \sin \theta)} \exp (i n \phi)
$$

and the pressure of the fluid layer when the plane wave excitation acts can be expressed as follow:

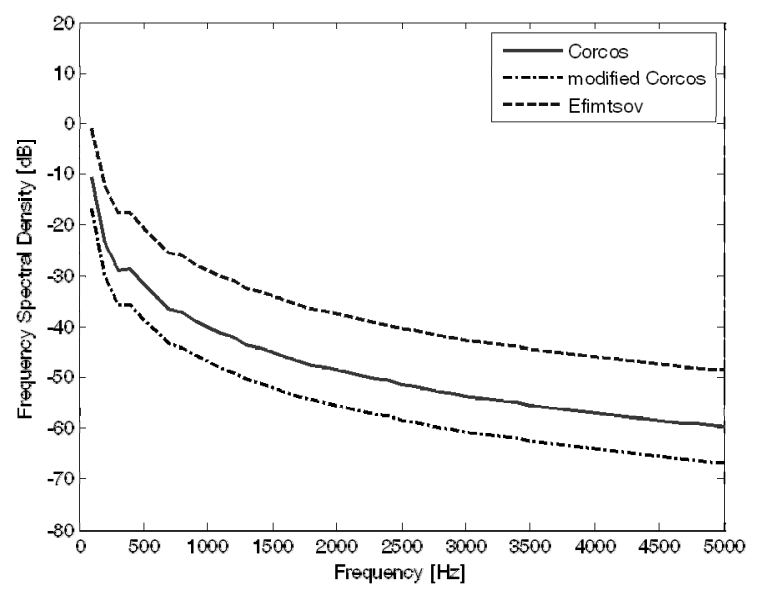

(a) Frequency spectral density.

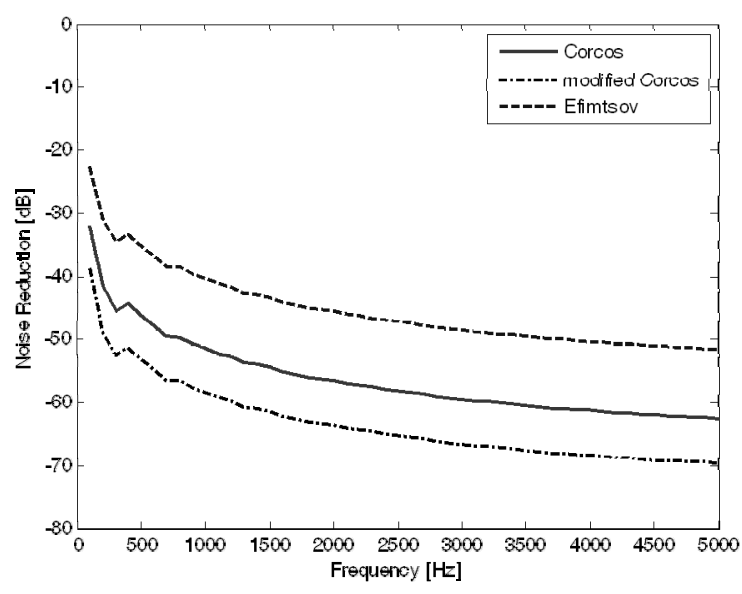

(b) Noise reduction.

Fig. 14 Comparisons for various turbulent wall pressure spectrum at the sensor position excited by turbulent flow. Free stream velocity is $10 \mathrm{~m} / \mathrm{s}$. 


$$
p(r, \alpha)=\sum_{n=-\infty}^{\infty}\left(A_{1} J_{|n|}(\gamma r)+A_{2} H_{|n|}(\gamma r)\right)
$$

The cylindrical model possessed a radius of $4 \mathrm{~m}$. The pressures were summed between -100 to 100 of the Bessel function order $n$. The results of the cylindrical model are very similar to the results of the planar model. However, the cylindrical model required over 200 times the amount of analysis time more than the planar model. Additionally, many turbulent flow wall pressure spectrum models are available for the planar surface (Ko, 1994; Efimtsov, 1982; Graham, 1997) (Fig. 14). Thus, the infinite planar model is appropriate for analysis of the HMS multi-layer system.

\section{CONCLUSION}

This paper presented an infinite planar analysis method that predicts the acoustic characteristics of an HMS multi-layer system. This method can be applied to the preliminary design stages of HMS multi-layer systems. The main excitations that influence the HMS of a submerged vehicle were classified into three excitation types: mechanical excitation, plane wave excitation, and turbulent flow excitation. Each excitation type was applied to various models for analysis. The stiffened multi-layer for mechanical excitation was developed and the general multi-layer model analysis for turbulent flow excitation was expended. Furthermore, the planar theory proved to be better than the cylindrical theory for evaluating the HMS multi-layer system.

This paper did not make a conclusion which model was the best. Because many conflicting goals are considered at the preliminary design stages. Noises from mechanical excitation and turbulent flow should be reduced, even though sensors should catch signals well from outside of the submerged vehicle. We expect that designers can adopt assistant systems to achieve the design goals if they know the acoustic characteristics of HMS multi-layer systems using infinite planar analysis method.

\section{ACKNOWLEDGEMENTS}

This research was supported by the Agency for Defense Development of Korea. We would like to thank them for their assistance.

\section{REFERENCES}

Corcos, G.M., 1964. The structure of the turbulent pressure field in boundary layer flows. Journal of Fluid Mechanics, 18 (3), pp.353-378.

Efimtsov, B.M., 1982. Characteristics of the field of turbulent wall pressure fluctuations at large Reynolds numbers. Soviet Physics-Acoustics, 28(4), pp.289-292.

Evseev, V.N., 1973. Sound radiation from an infinite plate with periodic inhomogeneities. Soviet Physics-Acoustics, 19(3), pp.226-229.

Graham, W.R., 1997. A comparison of models for the wavenumber-frequency spectrum of turbulent boundary layer pressures. Journal of Sound and Vibration, 206(4), pp.541-565.

Kang, M.W., Seo, Y.S., Shin, K.K. and Jeon, J.J., 2011. Acoustic performance study of FRP acoustic window. Proceeding of the Korean Society for Noise and Vibration Engineering Annual Spring Conference. Jeongseon, Korea, pp.285-290.

Ko, S.H. and Schloemer, H.H., 1989. Calculations of turbulent boundary layer pressure fluctuations transmitted into a viscoelastic layer. Journal of the Acoustical Society of America, 85(4), pp.1469-1477.

Ko, S.H. and Nuttall, A.H., 1991. Analytical evaluation of flush-mounted hydrophone array response to the Corcos turbulent wall pressure spectrum. Journal of the Acoustical Society of America, 90(1), pp.579-588.

Ko, S.H., 1994. The flush-mounted hydrophone array response to a modified Corcos turbulent wall pressure spectrum (analytical approach). Journal of the Acoustical Society of America, 96(2), pp.1198-1201.

Ko, S.H., Pyo, S.W. and Seong, W.J., 2001. Structure-Borne and Flow Noise Reductions: Methematical Modeling. Seoul : Seoul National University Press.

Mace, B.R., 1980. Sound radiation from a plate reinforced by two sets of parallel stiffeners. Journal of Sound and Vibration, 71(3), pp.435-441.

Skelton, E.A. and James, J.H., 1997. Theoretical Acoustics of Underwater Structures. London : Imperial College Press. 